\title{
TRIPLE-SYSTEMS AS TRANSFORMATIONS, AND THEIR PATHS
}

\section{AMONG TRIADS*}

BY

\section{H. S. WHITE}

A triple-system has been of interest as offering a puzzling problem in construction. When various methods of construction have given systems apparently different, means of comparison have to be devised. The group of substitutions that transform a triple-system into itself has been adopted as a convenient abstract mark or character for discrimination; since certainly two systems whose groups are abstractly different cannot belong to similar triplesystems. I propose here a more direct means of comparison of two triplesystems, a method which incidentally facilitates the discovery of the group. This method consists in regarding the triple-system itself as a symbol of operation, and deducing certain covariants of that operation. These covariants can be represented in some respects graphically. I here apply such graphic representation to the well-known triple-systems on thirteen elements; thirteen being the lowest number of elements for which there exist two dissimilar or incongruent triple-systems.

As in some other transformations, so here also the inverse problem of invariance presents difficulties, the question, namely, what relations between the invariants of two operations are sufficient to prove the equivalence of the operations themselves. In the case here presented, where the possible operations (triple-systems on thirteen elements) are only two in number, the answer is immediate and complete; but I do not attempt any generalization.

\section{Reciprocity or duality in a finite set mediated by a triple-system.}

Recall the definition of a triple-system. It consists of triads of the chosen elements, so numerous and so related that while every pair of elements (or dyad) occurs in some triad, no pair occurs in two triads. Thus if there are 13 elements, every element occurs with 6 pairs of others, and there are in all 26 triads.

It is precisely this property that fits the triple-system to be a transformer of

\footnotetext{
* Presented to the Society, September 11, 1912.
} 
dyads into single elements. Since every dyad occurs once and no more, this duality will be unique for dyads; but its inverse would transform a single element into 6 dyads. Let us consider solely the transformation of a dyad into an element, and the resulting transformation of triads into triads.-Every combination of three elements comprises three dyads; and when each of these becomes an element, the triad becomes a new triad. Thus if the triple system contain the three triads $124,135,236$, then it will transform the triad 123 into 456.

From 13 elements, 286 triads can be formed. Any proposed triple-system contains 26 of these, leaving 260 that we may call extraneous triads. Apply the triple-system to transform them all; the 26 in the system are all self dual, i. e., they are transformed into themselves, but the 260 extraneous triads go partly into extraneous, partly perhaps into triads of the system. All that are found to be produced by the transformation we may call derivative, all that are missing after the transformation we shall call primitive. By trial on the two types of triple-systems, Reiss's and NeTTo's, we find that the former leaves out 104 primitive triads, the latter 130 .

This distinction of primitive and derivative is obviously not affected if the triple-system and all extraneous triads be themselves subjected to any operation of substitution upon the 13 elements. Hence the distinction is invariant under the smaller group of substitutions which transform the triple-system into itself. We have then, under this group, three invariant sets of triads: those in the system, the extraneous-derivative triads, and the extraneousprimitives. Other subdivisions will appear soon.

Iterated duality. Trains of triads.

Under the dual transformation of a given triple-system $\Delta$, let a triad $t_{1}$ be converted into another triad $t_{2}$. Repeat the operation; let $t_{2}$ become $t_{3}$. Continue the iteration indefinitely. Since only 286 triads exist, one of two things must happen: either a triad of the system is reached, say $t_{k}$; or else a triad that has appeared already is repeated, say $t_{m+n} \equiv t_{m}$. In the former case the triad $t_{k}$ repeats forever, while in the latter case the train beginning from $t_{m}$ constitutes an ever recurring cycle. Both cases may be included in one if we will speak of the triads of the system as one-term cycles. We can formulate the statement that every triad that is primitive with respect to a given triple-system initiates a train terminating in a periodic cycle. Presumably any one cycle may have several initial primitive triads, though it is conceivable that it might be made up exclusively of derivatives. If there are in a train primitives and derivatives that do not recur in the terminal cycle, they may be classified as forming appendices; then every complete train will be made up of one recurrent cycle and all its appendices. In the two examples to be pre- 
sented there are appendices both short and long, some consisting of a single primitive triad, some of a primitive and seven derivatives.

The totality of the periodic cycles constitutes a system of triads, invariant under the automorphic group of the triple-system and also invariant under the dual transformation mediated by the triple-system itself.

The totality of complete trains (cycles with their appendices) constitutes a system of triads invariant under those substitutions on the 13 elements that transform the triple-system into itself, and covariant with the triple-system under the symmetric group on the 13 elements.

\section{The Reiss triple-system on 13 elements.}

The first to write down the triples of a system on 13 elements was Reiss, whose system may be arranged for convenience in the following rectangular array. Each element heads one column, and below are placed the 6 pairs of elements that occur with it in triads of the system.

\begin{tabular}{c|c|c|c|c|c|c|c|c|c|c|c|c}
\hline \hline 1 & 2 & 8 & 4 & 5 & 6 & 7 & 8 & 9 & $a$ & $b$ & $c$ & $d$ \\
\hline 23 & 13 & 12 & 56 & 46 & 45 & 26 & 15 & 16 & 59 & 47 & 14 & 17 \\
58 & 67 & 49 & 28 & 18 & 19 & 35 & 24 & 34 & 78 & 29 & 79 & 25 \\
69 & 48 & 57 & 39 & 37 & 27 & $1 d$ & $7 a$ & $5 a$ & 36 & 38 & 68 & 89 \\
$4 c$ & $5 d$ & $6 a$ & $1 c$ & $2 d$ & $3 a$ & $4 b$ & $9 d$ & $2 b$ & $1 b$ & $1 a$ & $2 a$ & $4 a$ \\
$7 d$ & $9 b$ & $8 b$ & $7 b$ & $9 a$ & $8 c$ & $8 a$ & $3 b$ & $7 c$ & $2 c$ & $5 c$ & $5 b$ & $6 b$ \\
$a b$ & $a c$ & $c d$ & $a d$ & $b c$ & $b d$ & $9 c$ & $6 c$ & $8 d$ & $4 d$ & $6 d$ & $3 d$ & $3 c$ \\
\hline
\end{tabular}

A triad extraneous to this system is for example 246. In column 2 we note the pair 48 and the pair 67, in column 4 the pair 65 , and have thus found the transformed triad 578. This in turn transforms into 31a, etc. The process is simple and not too tedious, and we find eight classes of trains, as follows:

I. Six classes terminating in triads of the system:

(1) Three with no appendix, $169,79 c$, and $89 d$. $1-1$

(2) Four with two single appendices. Example

$$
{ }_{4 a b}^{235} 17 d .>1-1
$$

(3) Six with a double appendix. Example,

$$
37 a 56814 c \text {. - - - }
$$

(4) Nine with double bifid appendix. Example,

$$
\begin{aligned}
& 46 b \\
& 89 a
\end{aligned} 57 d \quad 123 .>-1
$$

(5) One with single trifid appendix, viz., 
(6) Three with two appendices, one quadruple and one octuple bifid. Example:

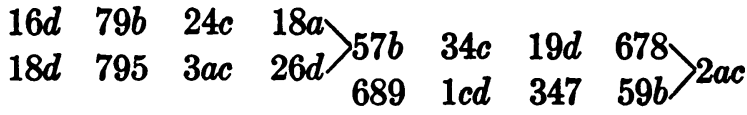

$$
\begin{aligned}
& -\longrightarrow-7>1 \text {. }
\end{aligned}
$$

II. Two classes terminating in cycles of period 6.

(7) Six whose appendices in order contain 1, 1, 1, 2, 2, 2 triads. Example: one whose initial primitives are $7 a d, 468,259,35 c, 3 a b, 134$.

(8) Six whose appendices in order contain 1, 2, 0, 1, 2, 0 triads. Example: one whose initial primitives are 457, 149, 12a, 279.

Graphs showing the order of succession of these latter two classes are the most noteworthy new result brought out by this method. For a rough sketch we may omit the elements of the triads, and denote each triad by a segment of a line, make a primitive end where a derivative begins, arrange the cycle as a regular hexagon, and insert an arrow to point the direction of derivation. Appendices fall outside, and their extreme segments are the primitives of the trains.

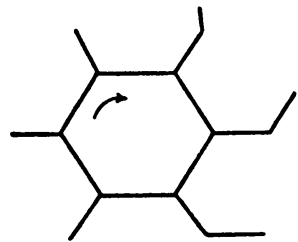

Class 7, 6 trains.

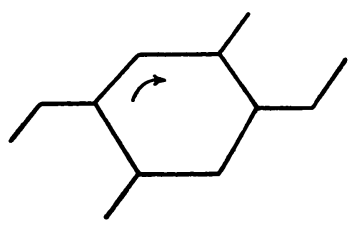

Class 8, 6 trains.

Netto's triple-system on 13 elements.

The second kind of triple-system on 13 elements is NETTo's, given here in the same tabulation as RErss's.

\begin{tabular}{c|c|c|c|c|c|c|c|c|c|c|c|c}
\hline \hline 1 & 2 & 8 & 4 & 5 & 6 & 7 & 8 & 9 & $a$ & $b$ & 0 & $d$ \\
\hline $2 b$ & $3 c$ & $4 d$ & 15 & 26 & 37 & 48 & 59 & $6 a$ & $7 b$ & $8 c$ & $9 d$ & $1 a$ \\
$a d$ & $1 b$ & $2 c$ & $3 d$ & 14 & 25 & 36 & 47 & 58 & 69 & $7 a$ & $8 b$ & $9 c$ \\
45 & 56 & 67 & 78 & 89 & $9 a$ & $a b$ & $b c$ & $a d$ & $1 d$ & 12 & 23 & 34 \\
79 & $8 a$ & $9 b$ & $a c$ & $b d$ & $1 c$ & $2 d$ & 13 & 24 & 35 & 46 & 57 & 68 \\
38 & 49 & $5 a$ & $6 b$ & $7 c$ & $8 d$ & 19 & $2 a$ & $3 b$ & $4 c$ & $5 d$ & 16 & 27 \\
$6 c$ & $7 d$ & 18 & 29 & $3 a$ & $4 b$ & $5 c$ & $6 d$ & 17 & 28 & 39 & $4 a$ & $5 b$ \\
\hline
\end{tabular}

When triads are transformed through this table, the resulting trains fall into 3 classes of 13 each. Two take the place of the first six of the Reiss case; and the entirely extraneous trains are a single class, all similar, instead of two sixes. 
Triads of the system terminate 26 .

(1) Thirteen have a double appendix. Example: 49b, 236, 57c. $--1-1$.

(2) Thirteen have three simple appendices.

Example:

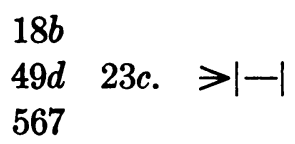

External triads form also a third class.

Class (3). Thirteen terminate in cycles of period 6, with appendices in order containing 1, 2, 1, 2,1, 2, triads. Example: one whose primitives are $7 c d, 17 b, 346,68 c, 59 a, 2 a c$.

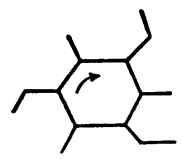

Class 3, 13 trains.

These graphs render the dissimilarity of the two systems obvious.

\section{The trains exhibit the groups.}

Substitutions on the 13 elements will transform every train into one precisely similar. Therefore any operation of the group that leaves a triple-system invariant can only change any particular train into itself, or into another of the same class. To find that group, then, we may seek first substitutions that transform into itself one of the largest trains, then those that transform it into each one in succession of its own class. Substitutions thus found must be tested on all trains of the class simultaneously, then upon the other classes, and only those retained which conserve every class. Or when found they may be tested directly upon the triple-system itself.

For the Netto $\Delta_{13}$ this is quite simple. Looking at the graphs of the third class, we surmise that each has cyclic substitutions into itself of period 3 , and that each may be transformed into any one of the other twelve-i. e., that these transformations, with the identity, constitute a sub-group of order 13. Barring a third conceivable style of change, this would give us a total group of order 39.

The other possibility is namely this, that some substitution on the elements might convert every triad contained in a train into itself; i. e., that the identity on triads might result from some substitution not the identity on the elements. The larger the train, the easier the inspection that discards this hypothesis; 
for any letter that is found in two triads having the other letters different must go over into itself if both triads are to go over into themselves. That conceivable third style of substitutions is found to be impossible in either set of trains.

We shall give special detailed attention to the group of the Reiss $\Delta_{13}$. For this purpose it will be shortest to use the six trains of Class 7. None of them has any substitution into itself; and we shall find that the requirement of changing the first into any other particular train determines the substitution completely. In writing these trains, let a bar at the left of a triad denote a primitive, and let a dash between two indicate that the one at the right is derived from that at the left through the Reiss $\Delta_{13}$. The six trains of Class 7 are the following, with appendices both above and below the line of the repeating cycle of six.

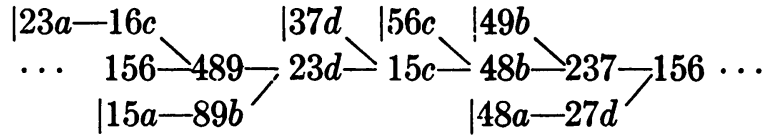

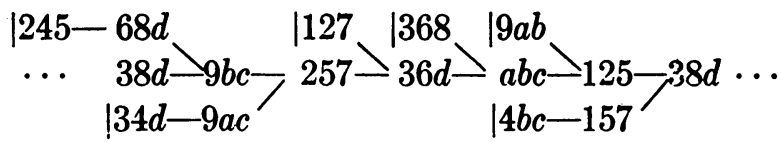

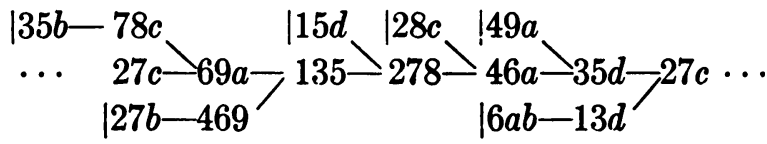

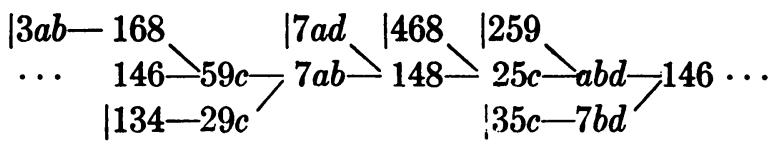

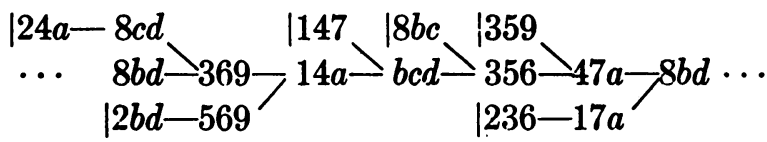

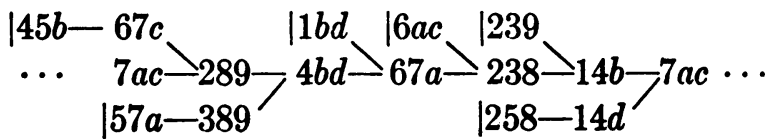

To transform the first of these trains into the second, for example, evidently we must transform each triad into one in the same vertical column, and indeed into the one lying in the corresponding horizontal line. In the second column from the left we see that 1 , occurring three times, must go over into $d$; similarly in the sixth column 4 must go over into $b$. Again in column two, lines one and two, 6 goes into 8 , and so $c, 5$ into 6,3 respectively; and in line three, $a$ into 4 . Exactly in the same way from column six we find that $b, 9,8$ go over into $a, 9, c$ 
respectively. This leaves for determination $2,3,7, d$. Of these, 2,7 in column four are seen to go over into 5,1 respectively, while the other two are found conveniently in column seven, where we have $3, d$ converted into 2,7 . The complete substitution is thus determined, as was to be proven. It is, condensed in usual form,

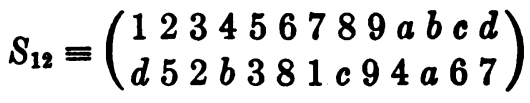

$$
\begin{aligned}
& \equiv(9)(1 d 7)(253)(4 b a)(68 c) \text {. }
\end{aligned}
$$

Indeed this has for a corollary the non-transformability of the second train into itself. Looking further, we verify that $S_{12}$ changes the third into the first, the second into the third, and permutes cyclically also the fourth, fifth, and sixth trains of this class. But when we once have the substitution in hand, it is shorter to prove it directly upon the tabular form of the $\Delta_{13}$ at once; and there we find the entire columns permuted with the same substitution as the elements.

It is necessary further only to find a substitution, $S_{14} \equiv(2 b)(3 a)(45)(7 d)(8 c)$, that converts the first train into the fourth of Class 7, and that with $S_{12}$ generates the group of order 6 . This being well known as the group of this $\Delta_{13}$, the method receives confirmation, and need not be carried out in further detail.

\section{Conclusion.}

Attention having been given hitherto chiefly to the construction of triplesystems, the time has come for new methods of comparison and description, as contribuing to facility in classification.

By the analogue of projections and linear transformations, applied under the concept of finite geometries, we are led to regard the triple-system as an operator. Like the quantic in symbolic algebra, it has an indefinitely extensive range of objects on which to act. In this sketch, we have applied it to triads, and found that the triple-system $\Delta_{13}$ operating on all triads of its elements arranges them in trains, most of which are characteristic of the system; and that especially the trains composed exclusively of triads extraneous to the system are an aid in the discovery of the group to which the system belongs. In the light of existing knowledge of the two species of $\Delta_{13}$ 's we can formulate theorems whose independent proof is not yet found. One example is sufficient:

THEorem: If any triple-system on 13 letters contains a triad not derivable, through the duality mediated by the system, from any extraneous triad, then the triple-system is of the Reiss species and possesses three such triads.

One may examine sets of 13 triads that are invariant under the group of the Netto triple-system. Of these there are but two, the primitives and first 
derivatives in the 13 trains of the first class. These two together constitute a second Netto triple-system, equivalent of course to the first This, with the recollection that the group arranges all triads in conjugate sets, makes evident the theorem:

Triple-systems of the Netto type on 13 elements occur in pairs, the two of a pair having the same group but having no triad in common. The triads in such a pair can combine by alternating thirteens to form in one way a second pair of triple-systems related to each other in the same way as the first pair. Of interest is the fact that if the two systems of one pair be employed to transform each other, the other pair appears; and a second transformation with the new systems restores the old in inverted order. 